\title{
Students' Learning Performance and Transitions in Different Learning Pathways to Higher Vocational Education
}

\author{
Harm Biemans ${ }^{1}$ (D) Hans Mariën ${ }^{2}$ - Erik Fleur ${ }^{3}$. \\ Hilde Tobi $^{1} \cdot$ Loek Nieuwenhuis $^{4,5} \cdot$ Piety Runhaar $^{1}$
}

Received: 10 September 2015 / Accepted: 6 March 2016 /

Published online: 30 March 2016

(C) The Author(s) 2016. This article is published with open access at Springerlink.com

\begin{abstract}
To improve students' transitions between successive educational levels, continuing learning pathways are being designed and implemented in many countries. This study was carried out to examine the effects of the Green Lycea (GL) as critical cases of continuing learning pathways in vocational education in The Netherlands. The GL were compared with a traditional pathway (pre-vocational secondary education and secondary vocational education offered as separate programmes) in terms of students' learning performance and transitions. GL students and comparable students following the regular pathway yielded the same mean final exam scores for four core subjects in the first phase of their educational programmes, despite the fact that GL students took this exam one year earlier than regular students because of the acceleration of the learning trajectory in the GL programme. Regarding the students' transitions, the GL and the traditional pathway yielded comparable percentages of students who either did or did not obtain a pre-vocational secondary education diploma; For the GL, however, the percentage of students who proceeded in secondary vocational education was higher. To conclude, it seems to be possible to improve students' transitions between
\end{abstract}

Harm Biemans

harm.biemans@wur.nl

1 Education and Competence Studies Group, Wageningen University, P.O. Box 8130, 6700 EW, Wageningen, The Netherlands

2 IVA Education, Tilburg University, Warandelaan 2, 5037 AB Tilburg, The Netherlands

3 Department of Education, Culture, and Science, Executive Agency for Education (DUO), Europaweg 2, 2711 AH Zoetermeer, The Netherlands

4 Research Centre Quality of Learning, HAN University of Applied Sciences, P.O. Box 30011, 6503 HN Nijmegen, The Netherlands

5 Welten Institute for Research on Learning, Teaching and Technology, Open University, P.O. Box 2960, 6401 DL Heerlen, The Netherlands 
successive educational levels (with their learning performance being an important precondition) through continuing learning pathways such as the GL. In this regard, GL design characteristics as described and examined in this study can be valuable ingredients for continuing learning pathways internationally as well.

Keywords Continuing learning pathways $\cdot$ Educational transitions $\cdot$ Learning performance $\cdot$ Vocational education and training $\cdot$ Green Lyceum

\section{Introduction}

Many countries explicitly strive to enhance the educational level of their inhabitants to be able to face the challenges of modern society. A central goal in their educational policy is that higher percentages of people should reach higher levels of the educational system (Keeley 2007; OECD 2010). This trend is also visible in The Netherlands, the country in which this particular study is carried out: One of the key elements of Dutch educational policy of the last decades is that percentages of students reaching and successfully finishing higher vocational education should increase (Dutch Educational Council 2014).

To enable more students to reach higher vocational education, it is crucial that boundaries between successive educational levels are reduced. In other words, students should be enabled to develop their knowledge, skills, and competencies (required not only for dealing with present and future professional core tasks but also for their own personal development from the perspective of further education, lifelong learning, employability, and citizenship; see also Biemans et al. 2004, 2009; Wesselink et al. 2010a, b; Sturing et al. 2011) in seamless pathways without artificial barriers between educational levels (Harris and Rainey 2012).

In practice, however, the transitions from one educational level to the next are often problematic for students (Biemans et al. 2013). Comparable transition problems between successive educational levels have been recognised internationally as well (e.g., Bradley 2008; Hoelscher et al. 2008; Jäppinen and Maunonen-Eskelinen 2012; Catterall et al. 2014). These transition problems endanger educational policy to enhance the general educational level and seem to require a curriculum design solution, i.e., the design and implementation of new continuing learning pathways (see also Biemans et al. 2013). Therefore, like in other countries, the Dutch government has stimulated the design of new continuing pathways (Dutch Ministry of Education, Culture, and Science 2005). The aim of these continuing pathways is to ensure curriculum continuity of successive educational programmes and, thus, to promote and streamline students' knowledge and skill acquisition and competence development.

The present study was designed to examine to what extent such continuing learning pathways in Dutch Vocational Education and Training (VET) improve students' transitions between successive educational levels (with their learning performance being an important precondition). Therefore, these continuing pathways to higher vocational education were compared with a more traditional pathway to higher vocational education in terms of the students' learning performance and their transitions through the respective educational programmes (or study careers - cf., Bloomer and Hodkinson 2000a, 2000b). 
In the next section, transition problems in VET are discussed and continuing learning pathways are presented as a possible solution for these transition problems. The Green Lycea (GL) are described as critical cases of such continuing pathways. Next, the method of the present study is presented, followed by the results of the research. The article concludes with a discussion of the findings and a future research agenda.

\section{Theoretical Background}

\section{Transition Problems in VET}

As described by Kuijpers (2014), the accessibility of educational systems benefits from optimal transitions between educational levels (from primary education until university education in general and in specific segments of the educational system like the VET stream in particular). Optimal transitions are important for students to be able to continuously develop their competencies and to build a successful study career, but also for school organisations representing successive educational levels, the labour market, and society as a whole. Unfortunately, reality appears to be different in many countries: Students' transition problems between successive educational levels have been documented extensively in the international scientific literature (e.g., Hoelscher et al. 2008; Harris and Rainey 2012; Jäppinen and Maunonen-Eskelinen 2012). For many students, the various educational programmes corresponding with successive educational levels represent separate worlds, which makes it difficult for them to link and integrate the content of these programmes (Biemans et al. 2013). As a consequence, transition periods may be stressful for many students (Wheelahan 2008; Catterall et al. 2014).

In this regard, The Netherlands are no exception to the international pattern: Transitions in the Dutch educational system appear to be problematic as well (at this point, it should be noted though that the Dutch system is characterised by a rather early selection of students between vocational and general trajectories - see also Fig. 1 and OECD 2010). Indicators for such problematic transitions are high student drop-out rates and very sharp selection criteria used by schools from an output point of view (or, in other words, to prevent high drop-out rates) (Dutch Educational Council 2014, in: Kuijpers 2014). Moreover, in many cases, the study

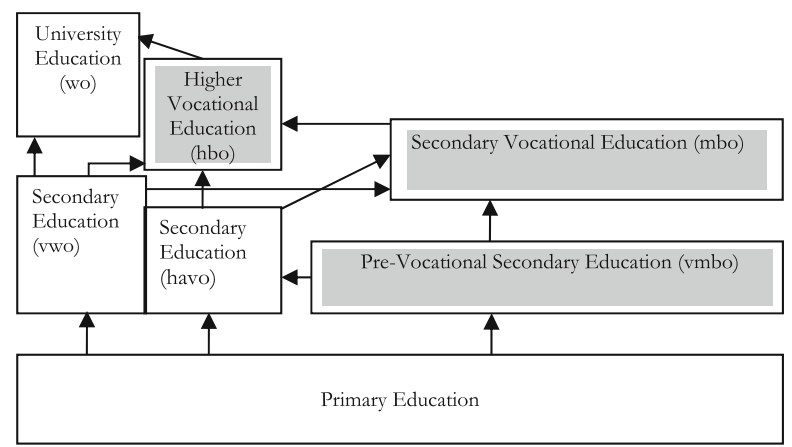

Fig. 1 The Dutch educational system (levels of the VET stream indicated in grey) 
career choices made by students themselves do not appear to be beneficial to them in the longer term as well (Winters 2012).

Students' transition problems between successive educational levels may have different causes (see e.g., Harris and Rainey 2012; Kuijpers 2014):

- Issues related to sharp student selection for and restricted admission to follow-up educational programmes may play a role;

- Contents, didactics, and/or learning environments of successive educational programmes may not be attuned because of insufficient cooperation between the respective educational organisations (Jäppinen and Maunonen-Eskelinen 2012) and different legislation for successive educational (sub)systems;

- Students' cognitive levels and/or study skills may not meet the required level for the follow-up programme;

- Students' motivation for a follow-up programme (or for education in general) may be lacking;

- Students may not have clear perceptions and expectations of possible follow-up programmes and/or clear ideas about their own desired future jobs or careers;

- Career orientation and guidance may be insufficient.

This phenomenon of problematic transitions is clearly visible in the VET system in The Netherlands (Biemans et al. 2013), which consists of three levels: pre-vocational secondary education (vmbo), secondary vocational education (mbo), and higher vocational education (hbo) (see Fig. 1).

In the transitions to the upper study years of pre-vocational secondary education (vmbo) and to the first study year of secondary vocational education (mbo), students are required to choose for a particular vocational sector. From the beginning of the $21 \mathrm{st}$ century, numbers of students who have switched from one sector in vmbo to another sector in mbo have increased significantly ever since (Kuijpers 2014). This phenomenon happens to be true for all educational sectors, although, in particular sectors, students are more loyal to the sector of their original choice than in other sectors (see also Harris et al. 2005). For the technical education sector, the percentages of students who stuck to their original sector choice while going from vmbo to mbo appeared to be the highest (from $90 \%$ in 2004-2006 to $71 \%$ in 2010-2011). On the other hand, for the agricultural or life sciences (or 'green') education sector, the percentages of internal transitions from 'green' vmbo to 'green' mbo were the lowest (from $60 \%$ in 2000 to only $29 \%$ in 2010-2011) although the absolute numbers of students entering 'green' vmbo have increased ever since, which leads to a relatively stable total number of students going from 'green' vmbo to 'green' mbo (see also Van den Berg 2013).

Another related issue are the transition problems many students experience between secondary vocational education (mbo) and higher vocational education (hbo). In the Dutch educational system, there are suitable, related pathways in hbo for almost every mbo sector. One could assume that this would ensure smooth student transitions between mbo and hbo. This, however, is not true in many cases. After completion of their mbo programmes, many students decide to look for a job on the labour market (which might actually be a good option for them) and not to proceed with their educational careers and to enhance their educational levels. Moreover, for the students who do decide to choose an hbo programme after receiving their mbo diploma, the 
drop-out rates in the first study years are relatively high. This trend is clearly visible in all educational sectors, including the 'green' education sector (AOC Council 2012). Higher vocational education access and transition problems corresponding with different educational pathways are also an issue internationally (see e.g., Gorard et al. 2006; Bathmaker and Thomas 2009; Jäppinen and Maunonen-Eskelinen 2012; Catterall et al. 2014).

These relatively low internal transition percentages from vmbo to mbo and from mbo to hbo make the 'green' education sector, which is the specific educational sector under study in this manuscript, very interesting from a transition problem perspective. Like other educational sectors in The Netherlands, the 'green' education sector has formulated specific ambitions to reduce the transition problems between the successive educational levels. One of these ambitions is to enhance the internal transition percentage from 'green' vmbo to 'green' mbo from the current less than $30 \%$ to $40 \%$ (AOC Council 2012).

\section{Continuing Learning Pathways as Possible Solution for Transition Problems}

The above-mentioned transition problems that many students experience between successive educational levels prevent them from reaching higher educational levels and, thus, endanger national educational policy to enhance percentages of students successfully finishing higher vocational education. Several possible solutions to tackle this problem have been proposed in the literature.

One possible solution is to promote the quality and intensity of career orientation and guidance for students in schools: Several researchers claim that career orientation and guidance should require more attention in schools through conversations between students and teachers and practical coaches to prepare the students for the crucial decisions to be made related to transitions between (and within) educational levels (e.g., Winters 2012). In their opinion, it is crucial to discuss different study and job perspectives together with (individual) students in relation to their own preferences and abilities to make them more aware of various options they have, to assist them in making choices for a particular educational programme and/or vocational sector based on argumentation and personal preferences, and to motivate them to follow the educational route of their choice. In order to have a significant effect on students' study careers, career orientation and guidance should take place on a regular basis and be part of a 'coherent architecture' of the students' learning environment (Kuijpers 2014). In this regard, based on research on the study careers of vmbo students until they are enrolled in mbo programmes, Winters (2012) showed that, career conversations have a consistent positive effect (next to age) on students' career competencies and choices.

Career orientation and guidance as such, however, do not solve problems of disconnectivity with respect to the content of educational programmes of successive levels in the educational system. As mentioned in the Introduction section, this seems to require a curriculum design solution, i.e., the design and implementation of new continuing learning pathways.

Continuing learning pathways can be defined as "sequential educational programmes combined into a new educational programme: continuing pathways are characterised by curriculum continuity in particular competence areas or subjects 
lasting several years, and encompassing more than one qualification level" (e.g., vmbo and mbo) (Biemans et al. 2013, p. 109). Through such continuing learning pathways, these new educational programmes are assumed to build up learning in a more strongly linked and integrated way than the particular successive but separate educational programmes since students' knowledge and skill acquisition and competence development are streamlined throughout the whole programme (cf. Brockmann et al. 2008; Clarke and Winch 2007; Putnam and Borko 2000).

\section{The Green Lycea as Critical Cases of Continuing Pathways}

Prominent examples of continuing pathways in Dutch VET in the agricultural (or 'green') education domain are the 'Green Lycea' (GL), covering vmbo and mbo levels in five or six (instead of the regular seven, i.e., four vmbo and three mbo) years and leading to a 4/5 qualification level in the European Qualification Framework (European Commission 2008). The first GL started in the years 2007 and 2008 as experiments of continuing learning pathways in Dutch vocational education with relatively small student numbers in 5 schools in 3 different VET institutes. In the school year 20142015, GL were offered by 12 schools of 6 VET institutes spread over The Netherlands to more than 1000 students, which is a substantial number in the 'green' education sector. GL students obtain a separate prevocational (vmbo) diploma in addition to the vocational (mbo) diploma at the end of the trajectory. The GL are specifically designed for students with cognitive abilities to reach the hbo level (indication: 'havo'/vwo' entry level; see Fig. 1) combined with a preference for practical assignments. Traditionally, these students would either follow a regular vmbo programme and a mbo programme before entering hbo or they would follow a pathway through general secondary education, especially havo (see Fig. 1) (see also Van den Berg 2013).

The central idea behind the development of the GL is that they would suit this specific target group better because the GL offer many possibilities for assignments related to vocational practices (as opposed to general secondary education) (Billett 2001; Griffiths and Guile 2003) and because the theoretical level is relatively high and the educational programme is accelerated (as compared with the combination of regular vmbo and mbo programmes). In other words, to meet the characteristics of this specific student target group, the GL combine elements of both traditional alternative pathways. The GL are specifically designed with the aim to prepare students for higher vocational education (hbo): during their GL period, students are supposed to develop the knowledge, skills, and competencies they need to make a good start in their successive hbo programmes.

One of the most essential characteristics of continuing pathways is the true integration of the content of the respective educational programmes. Biemans et al. (2013) compared two different GL variants in terms of the students' learning performance and satisfaction: One GL variant in which vmbo and mbo elements were truly integrated in the sense that mbo elements were introduced in the vmbo programme and one GL variant in which vmbo and mbo programmes were accelerated but still separated. The integrated GL variant turned out to be more effective that the separated GL variant: Students in the integrated GL type had higher scores on school wellbeing, school satisfaction, and motivation for school than students in the separated GL type and their learning performance in the first two study years stayed on the same level, while decreasing in the separated GL type. Apparently, it paid off to build a whole new, 
challenging GL curriculum integrating vmbo and mbo elements for this specific target group, instead of just reducing the overlap and accelerating the original vmbo and mbo programmes. Moreover, the integrated GL variant appeared to have more characteristics of powerful learning environments (more powerful elements with respect to learning content, structure and range, learning environment, and processing activities and broader guidance in the beginning of the educational programme) than the separated GL variant (see for more information on characteristics of powerful learning environments e.g., De Bruijn and Leeman 2011; Koopman et al. 2011). For this reason, all GL have now adopted an educational model that is characterised by integration of vmbo and mbo elements.

Compared with the traditional pathways mentioned above, the GL are typified by the following design characteristics, which are meant to tackle possible causes for transition problems as described in the section 'Transition Problems in VET' and to promote student learning performance (see also Biemans et al. 2013):

- Sharp selection of a specific category of students who combine a relatively high cognitive level with affinity for practical assignments in authentic settings (see also Jäppinen and Maunonen-Eskelinen 2012);

- Acceleration of the learning trajectory (increasing learning pace by shortening the learning pathway; see also Jäppinen and Maunonen-Eskelinen 2012);

- Integration of the respective educational (vmbo and mbo) programmes;

- Theoretical level attuned to higher vocational education;

- Tailor-made, adaptive education based on the talents and interests of individual students (see Biemans et al. 2013;

- Specific attention for development of study skills needed in higher vocational education (e.g., planning, collaboration, project work, entrepreneurial and research competence);

- Educational orientation on professional practice;

- Broad orientation on higher vocational education sectors (not only the 'green' domain but also other professional domains);

- Systematic attention for career orientation and guidance (see also Kuijpers 2014).

During the last couple of years, several other experiments have started in other sectors of Dutch vocational education as well but these have not been evaluated or researched yet. These other cases share many design characteristics with the GL, e.g., acceleration of the learning trajectory, integration of the respective educational (vmbo and mbo) programmes, specific attention for development of study skills, and systematic attention for career orientation and guidance. In other words, when it comes to design characteristics, the GL are highly representative for these other pathways and can be considered as a critical case.

\section{Aim of the Study and Research Questions}

As mentioned above, the aim of the present study was to examine whether it was possible to improve students' transitions between successive educational levels (with their learning performance being an important precondition) through continuing learning pathways such as the GL. This was done by studying the joint effect of the design 
characteristics mentioned above, as represented in the GL. Therefore, the GL were compared with a more traditional pathway (regular vmbo-mbo) to higher vocational education in terms of students' learning performance and their transitions through the respective educational programmes. This study focused on the first phase of the GL learning trajectory, which students are supposed to conclude with a prevocational (vmbo) diploma (the second phase of their educational programme (mbo) was not taken into account since substantial data from students in the second GL phase were not available yet). Moreover, the various GL institutes were compared to check whether there were differences in terms of learning performance and transitions of the students.

In the present study, the following specific research questions were formulated:

1. Do GL students and regular vmbo students differ in terms of learning performance (i.e., vmbo final exam scores)?

2. Do the various GL institutes differ in terms of students' learning performance?

3. Do GL and regular vmbo programmes differ in terms of percentages of students who obtain a vmbo diploma?

4. Do the various GL institutes differ in terms of percentages of students who obtain a vmbo diploma?

5. Do GL and regular vmbo programmes differ in terms of percentages of students who proceed with a mbo programme after obtaining their vmbo diploma?

6. Do the various GL institutes differ in terms of percentages of students who proceed with a mbo programme after obtaining their vmbo diploma?

\section{Method}

\section{Participants and Design}

To examine the first two research questions, the group consisting of all 165 GL students who had passed their vmbo exam in the school years 2012-2013 and 2013-2014, as registered in a national student database, was taken as the starting point. These students came from seven schools of three different Dutch 'green' VET institutes (the first three institutes that had started a GL programme already in 2007 or 2008).

Next, a control group of regular vmbo students was composed that resembled the above-mentioned group of GL students as much as possible in terms of the following student characteristics: general cognitive ability test score (based on the same standardised test taken by the students in primary education, or, in other words, their cognitive starting level at the beginning of their GL or vmbo trajectory), gender, and year of examination (2013 or 2014). The selection of the members of the control group was done in the following steps: First, individual regular vmbo students from the same institutes who matched individual GL students in terms of the student characteristics mentioned above were selected from the national student database. Then, comparable regular vmbo students from schools from the same municipality, from the same region, and from the same province were identified in the national student database and selected. This procedure resulted in a control group of regular vmbo students (in fact the top segment of regular vmbo students in terms of general cognitive ability test scores) that was comparable to the group of GL students (see Table 1). 
Table 1 Composition of the group of GL students and the control group of regular vmbo students in terms of general cognitive ability test score (categories: $<537$; 537-540; 541-544; 545-550 -which is the maximum score-; no score available), gender (Male; Female), and year of examination $(2013 ; 2014)$

\begin{tabular}{|c|c|c|c|c|c|}
\hline \multirow[b]{3}{*}{ General cognitive ability test score } & \multirow[b]{3}{*}{ Gender } & \multicolumn{2}{|l|}{ GL } & \multicolumn{2}{|c|}{ Regular vmbo } \\
\hline & & \multicolumn{2}{|c|}{ Year of examination } & \multicolumn{2}{|c|}{ Year of examination } \\
\hline & & 2013 & 2014 & 2013 & 2014 \\
\hline \multirow[t]{2}{*}{$<537$} & M & 7 & 14 & 7 & 14 \\
\hline & $\mathrm{F}$ & 5 & 10 & 5 & 10 \\
\hline \multirow[t]{2}{*}{$537-540$} & M & 5 & 17 & 5 & 17 \\
\hline & $\mathrm{F}$ & 6 & 16 & 6 & 16 \\
\hline \multirow[t]{2}{*}{$541-544$} & M & 5 & 20 & 5 & 20 \\
\hline & $\mathrm{F}$ & 4 & 10 & 4 & 10 \\
\hline \multirow[t]{2}{*}{$545-550$} & M & 6 & 9 & 6 & 9 \\
\hline & $\mathrm{F}$ & 4 & 7 & 4 & 7 \\
\hline \multirow[t]{2}{*}{ No score known } & M & 5 & 6 & 5 & 6 \\
\hline & $\mathrm{F}$ & 4 & 5 & 4 & 5 \\
\hline Total & & 51 & 114 & 51 & 114 \\
\hline
\end{tabular}

In the data analyses related to the first two research questions, learning performance data of 280 students were included (142 GL and 138 regular vmbo students; 157 male and 123 female students). Not all students could be included in the analyses because not for all students a general cognitive ability test score was available in the national student database (and general cognitive ability test score was included in the analyses as a covariate) and not all students had vmbo exam scores for all relevant subjects (see next section).

To examine the remaining four research questions regarding students' transitions, the group consisting of all 217 students who had started a GL programme in the years 2009 and 2010, as registered in the same national student database, was taken as the starting point. These students came from the same seven schools of three different Dutch 'green' VET institutes as the GL group identified to answer the first two research questions. GL student cohorts 2007 and 2008 were not included in the analysis because of the small numbers.

Next, a control group of 217 regular vmbo students was composed that resembled the above-mentioned group of GL students as much as possible in terms of the following student characteristics: general cognitive ability test score (based on the same standardised test taken by the students in primary education), gender, and first study year (2009 or 2010). The procedure for composing this control group was identical to the procedure used to compose the previous control group.

\section{Dependent Variables}

To measure students' learning performance, their national vmbo final exam scores for the following four subjects were taken (these exams were identical for GL and regular vmbo students): Dutch language, English language, Mathematics, and Biology. These 
subjects can be regarded as core subjects in the GL and vmbo curricula. At this point, it should be noted that the final vmbo exam as taken by GL and vmbo students includes other subjects as well, but these are not compulsory or selected by a vast majority of students. Therefore, these other subjects could not be taken into account in the analysis of students' learning performance.

The other dependent variables were related to students' transitions. With respect to the third and fourth research question, percentages of students who either did or did not obtain a vmbo diploma (in or before the study year 2014-2015) were calculated for the GL group and the control group of regular vmbo students. Regarding the fifth and sixth research question, percentages of students who either 1) did not proceed with a followup educational programme after obtaining their vmbo diploma, or 2) did proceed with a mbo programme, or 3) did proceed with general secondary education (havo) were calculated for both groups. At this point, it should be noted that the vmbo programme is primarily intended as a preparation for further vocational education (mbo - option 2). The options 1 and 3 thus imply that students do not proceed with vocational education (at least, not in the short term).

\section{Data Analysis}

To compare the learning performance of the GL students and the regular vmbo students, a MANCOVA with the 4 exam scores (DU, ENG, MATH, and BIO) as dependent variables, the factors Group (levels: GL vs. Regular vmbo) and Gender (levels: Male vs. Female), and the covariate General cognitive ability test score was carried out. Before MANCOVA, performance data were checked for (multivariate) normality and no unusual or worrisome patterns were detected.

Next, to examine possible differences between the various GL institutes in terms of students' learning performance, a MANCOVA with the 4 exam scores (DU, ENG, $\mathrm{MATH}$, and $\mathrm{BIO}$ ) as dependent variables, the factors GL Institute (levels: 3 different GL institutes) and Gender (levels: Male vs. Female), and the covariate General cognitive ability test score was done.

To answer the last four research questions, percentages of students from the GL group and the control group (Regular vmbo) were compared through Chi-Square tests (either Mantel-Haenszel or Pearson) using a Crosstabs (SPSS Statistics - Version 22) procedure. Non-parametric statistics were used to answer these research questions because of the (nominal) measurement level of the data resulting in percentages.

\section{Results}

No significant differences in learning performance (i.e., mean vmbo final exam scores) were found between GL students and comparable regular vmbo students: The main effect of the factor Group was not significant $(\mathrm{F}(4,269)=0.67 ; p=0.61)$ (see also Table 2). Moreover, no significant interaction effects between Group and Gender and/or General cognitive ability test score could be determined. Not surprisingly, General cognitive ability test score appeared to be a significant predictor of vmbo final exam score $(\mathrm{F}(4,269)=10.82 ; p \leq 0.001)$ (the Pearson correlation coefficient of the relation between ability test score and mean exam score was $0.36(p \leq 0.01)$. 
Table 2 Mean vmbo final exam scores of GL students and comparable regular vmbo students for the subjects Dutch language (DU), English language (ENG), Mathematics (MATH), and Biology (BIO)

\begin{tabular}{lcccc}
\hline & DU & ENG & MATH & BIO \\
\hline GL & 6.39 & 6.34 & 6.63 & 6.65 \\
Regular vmbo & 6.53 & 6.69 & 6.76 & 6.60 \\
\hline
\end{tabular}

No significant differences in learning performance were found between students from the three GL institutes either: The main effect of the factor Group was not significant $(\mathrm{F}(8,256)=0.60 ; p=0.78)$ (see also Table 3). Moreover, no significant interaction effects between GL Institute and Gender and/or General cognitive ability test score could be determined. Again, General cognitive ability test score appeared to be a significant predictor of vmbo final exam score $(\mathrm{F}(4,127)=3.50 ; p \leq 0.01)$.

With respect to the third research question, there were no indications for differences between GL and regular vmbo programmes in percentages of students who either did or did not obtain a vmbo diploma $\left(\mathrm{X}^{2}(1)=0.13 ; p=0.72\right)$ (see also Table 4). Of the GL students, $85.3 \%$ of the students obtained their vmbo diploma as part of the GL programme and $12.4 \%$ of the students obtained their vmbo diploma after leaving the GL programme and switching to the regular vmbo pathway ( $2.3 \%$ of the GL students did not obtain a vmbo diploma at all).

There were no indications either for differences between the various GL institutes in percentages of students who either did or did not obtain a vmbo diploma $\left(X^{2}(2)=0.75\right.$; $p=0.69$ ) (see also Table 5).

Regarding the fifth research question, GL and regular vmbo programmes appeared to differ in percentages of students who either 1) did not proceed with a follow-up educational programme after obtaining their vmbo diploma (e.g., these students could have taken a job, have received unemployment benefit, have emigrated, or have gone to adult training), or 2) did proceed with a mbo programme, or 3) did proceed with general secondary education $\left(\mathrm{X}^{2}(2)=14.02 ; p \leq 0.001\right)$. For the GL, the percentage of students who proceeded with a mbo programme (after obtaining their vmbo diploma) was higher. For the regular vmbo programmes, the percentage of students who proceeded with general secondary education (and left vocational education) was higher (see also Table 6).

There were no indications that the various GL institutes differed in percentages of students who proceed with a mbo programme (either as part of the GL programme or as part of the regular mbo pathway after leaving GL) after obtaining their vmbo diploma $\left(\mathrm{X}^{2}(6)=10.49 ; p=0.11\right)($ see also Table 7$)$.

Table 3 Mean vmbo final exam scores of students from the three GL institutes for the subjects Dutch language (DU), English language (ENG), Mathematics (MATH), and Biology (BIO)

\begin{tabular}{lllll}
\hline & DU & ENG & MATH & BIO \\
\hline Institute 1 & 6.49 & 6.41 & 6.51 & 6.85 \\
Institute 2 & 6.29 & 6.12 & 7.07 & 6.63 \\
Institute 3 & 6.32 & 6.64 & 6.03 & 6.20 \\
\hline
\end{tabular}


Table 4 Percentages of students who either did or did not obtain a vmbo diploma for the GL and the regular vmbo programmes for the cohorts 2009 and 2010 and the combined cohorts (total)

\begin{tabular}{|c|c|c|c|c|}
\hline & & \multicolumn{2}{|c|}{ Vmbo diploma } & \multirow[t]{2}{*}{ Total } \\
\hline & & No & Yes & \\
\hline \multirow[t]{3}{*}{2009} & GL & 1.1 & 98.9 & 100.0 \\
\hline & Regular vmbo & 1.1 & 98.9 & 100.0 \\
\hline & Total & 1.1 & 98.9 & 100.0 \\
\hline \multirow[t]{3}{*}{2010} & GL & 3.2 & 96.8 & 100.0 \\
\hline & Regular vmbo & 1.6 & 98.4 & 100.0 \\
\hline & Total & 2.4 & 97.6 & 100.0 \\
\hline \multirow[t]{3}{*}{ Total } & GL & 2.3 & 97.7 & 100.0 \\
\hline & Regular vmbo & 1.4 & 98.6 & 100.0 \\
\hline & Total & 1.8 & 98.2 & 100.0 \\
\hline
\end{tabular}

Table 5 Percentages of GL students who either did or did not obtain a vmbo diploma for the various GL institutes

\begin{tabular}{llll}
\hline & \multicolumn{2}{c}{ Vmbo diploma } & Total \\
\cline { 2 - 3 } & No & Yes & \\
\hline Institute 1 & 2.1 & 97.9 & 100.0 \\
Institute 2 & 1.4 & 98.6 & 100.0 \\
Institute 3 & 3.8 & 96.2 & 100.0 \\
Total & 2.3 & 97.7 & 100.0 \\
\hline
\end{tabular}

Table 6 Percentages of students who either did not proceed with a follow-up educational programme after obtaining their vmbo diploma, or did proceed with a mbo programme, or did proceed with general secondary education (havo) for the GL and regular vmbo programmes

\begin{tabular}{llccc}
\hline & \multicolumn{2}{l}{ Educational programme after obtaining vmbo diploma } & Total \\
\cline { 2 - 4 } & None & Mbo & Havo & \\
\hline GL & 2.0 & 96.6 & 1.5 & 100.0 \\
Regular vmbo & 0.5 & 90.1 & 9.4 & 100.0 \\
Total & 1.2 & 93.3 & 5.4 & 100.0 \\
\hline
\end{tabular}


Table 7 Percentages of GL students who either did not proceed with a follow-up educational programme after obtaining their vmbo diploma, or did proceed with a mbo programme (either as part of the GL programme or as part of the regular mbo pathway after leaving GL), or did proceed with general secondary education (havo) for the various GL institutes

\begin{tabular}{llllll}
\hline & \multicolumn{2}{l}{ Educational programme after obtaining vmbo diploma } & Total \\
\cline { 2 - 4 } & None & Mbo (in GL) & Mbo (regular) & Havo & \\
\hline Institute 1 & 1.2 & 83.7 & 15.1 & & 100.0 \\
Institute 2 & & 77.9 & 19.1 & 2.9 & 100.0 \\
Institute 3 & 6.0 & 68.0 & 24.0 & 2.0 & 100.0 \\
Total & 2.0 & 77.9 & 18.6 & 1.5 & 100.0 \\
\hline
\end{tabular}

\section{Conclusions and Discussion}

The aim of the present study was to examine the effects of innovative examples of continuing learning pathways in vocational education in The Netherlands: The 'Green Lycea' (GL) with their specific design characteristics as described in the section 'The Green Lycea as Critical Cases of Continuing Pathways'. GL are designed and implemented to tackle students' transition problems between successive educational levels of the vocational education stream. Our study shows that GL, which are one year shorter in time, lead to similar learning performance compared to the traditional pathway. Moreover, for the GL, the percentage of students who proceeded in secondary vocational education was higher than for the traditional pathway. Both findings will be elaborated on in more detail below.

As shown in the present study, GL students and comparable vmbo students (i.e., the upper segment of regular vmbo students when their general cognitive ability level is concerned) did not appear to differ in learning performance in terms of mean vmbo final exam scores for the four selected subjects, which constitute the core of their respective educational programmes. At this point, however, it should be noted that, on average, GL students take their vmbo final exam in most subjects one year earlier than regular vmbo students (after three years instead of four years) because of the acceleration of the vmbo learning trajectory in the GL programme. Thus, the GL programme required one year less to cover the vmbo topics. The only exceptions in this regard are the subjects Biology at Institute 1 and Mathematics at Institute 2, for which the vmbo final exam is in the fourth year. Students from the various GL institutes did not appear to differ in terms of learning performance in the first phase of the learning pathway either. In other words, the GL offered by the various institutes did lead to comparable student learning performance across subjects (cf. Biemans et al. 2013).

Therefore, the main conclusion regarding students' learning performance is that GL students appeared to reach comparable performance levels as regular vmbo students in a shorter time period (at least, in the first phase of the learning pathway, which was examined in the present study). Whether and how this benefit continues into the next phase of their learning pathway warrants further investigation. In this regard, the acceleration in the GL seemed to be beneficial for these students with relatively high cognitive ability levels, which seems to prove the added value of these continuing learning pathways with their specific design characteristics. Corresponding with the general idea behind the development of the GL, these pathways seemed to suit this specific target group better and 
to offer more cognitive challenges than the regular vmbo trajectory given the relatively high cognitive level of the GL and the acceleration of the educational programme as explained in the section 'Theoretical Background'. In this respect, Jäppinen and Maunonen-Eskelinen (2012) also stressed the importance of entrance examinations and of shortening students' learning pathways as characteristics of effective continuing learning pathways (see also Hoelscher et al. 2008; Harris and Rainey 2012).

Regarding the students' transitions, GL and regular vmbo programmes did not appear to differ in terms of percentages of students who either did or did not obtain a vmbo diploma. On average only $2.3 \%$ of the GL students (again, there were no differences between the various GL institutes) and $1.4 \%$ of comparable vmbo students did not manage to obtain a vmbo diploma at all. At this point, however, it should be noted again that only the upper segment of regular vmbo students with respect to general cognitive ability level (comparable with the GL students) was included in this study. When the whole population of regular vmbo students would have been considered, percentages of students without a vmbo diploma would have been higher (cf. Van den Berg 2013). Obviously, obtaining a vmbo diploma is a major condition for being able to proceed in the VET stream and to prevent transition problems.

As mentioned in the Results section, of the GL students, $85.3 \%$ of the students obtained their vmbo diploma as part of the GL programme and $12.4 \%$ of the students obtained their vmbo diploma after leaving the GL programme and switching to the regular vmbo pathway. Apparently, the GL pathway is not equally suited for all students who started this educational programme. This shows the necessity of offering the option to students to switch to the regular vmbo pathway when the GL is too demanding and complicated for them. Therefore, students' progress should be monitored continuously and be discussed with them on a regular basis and, if necessary, a joint decision should made to switch to regular vmbo. Switching to regular vmbo did not seem to lead to additional difficulties for them in the sense that the majority of the former GL students still obtained their vmbo diploma.

When students have obtained their vmbo diploma in the Dutch VET system, there are several options: Students might not proceed with a follow-up educational programme, they might proceed with a mbo programme, or they might proceed with general secondary education (havo) (see also Fig. 1). GL students and comparable regular vmbo students appeared to differ in this respect: As mentioned in the Results section, for the GL, the percentage of students who proceeded with a mbo programme was higher (again, no differences were found between the various GL institutes). On the other hand, for the control group of regular vmbo programmes, the percentage of students who proceeded with general secondary education (and left vocational education) was higher. In other words, the percentage of students who stayed in the VET stream was higher for GL students than for regular vmbo students (this difference in students leaving the VET stream would have been even higher if a representative sample of regular vmbo students would have been concerned and not only the top segment of vmbo students in terms of general cognitive ability level - cf. Van den Berg 2013), which is an indication that transition problems between vmbo and mbo were reduced (cf. Hoelscher et al. 2008; Harris and Rainey 2012). This finding was confirmed by Van den Berg (2013), who reported lower drop-out rates for the GL trajectories and less transition to other vocational education sectors than for the more traditional vmbo-mbo routes.

To conclude, based on the results of this study, it seems to be possible to improve students' transitions between successive educational levels (with their learning performance being an important precondition) through continuing learning pathways such as the GL: Continuing learning pathways as the GL with their specific design characteristics seem to 
be more effective in terms of students' learning performance than the traditional vmbo pathway and to have the potential to reduce transition problems between various levels of the VET stream for this specific group of students (students with cognitive abilities to reach the hbo level combined with a preference for practical assignments) (see also Biemans et al. 2013). In other words, the GL characteristics (as described in the section 'The Green Lycea as Critical Cases of Continuing Pathways'), which were meant to tackle possible causes for transition problems and to promote student learning performance, seem to be effective in this regard. Comparable continuing learning pathways could be an option to tackle transition problems between various educational levels, to foster student participation, progression, and success in vocational learning (cf. Hayward 2006) and to promote access to higher vocational education in other countries as well (see also Bradley 2008; Gorard et al. 2006; Watson 2006; Jäppinen and Maunonen-Eskelinen 2012).

In this respect, Catterall et al. (2014, p. 242) stressed the importance of providing "timely information about differences between institutional and learner expectations, workload, administrative processes, and learning practices" to facilitate students' learning journey from one educational level to the next and to prevent transition problems from happening. In our opinion, however, students should be explicitly prepared in their educational pathways for what they can expect when they enter the next educational level in order to reduce the gap between student expectations and reality (Leese 2010), to overcome differences between the various levels in the learning and teaching context (Krause and Coates 2008) and to improve their learning performance. This actual preparation of students goes beyond the provision of timely information: In the GL, this preparation is realized e.g. through integration of the respective educational programmes, specific attention for the development of study skills needed at the next educational level, and systematic attention for career orientation and guidance (see also Kuijpers 2014). This approach, which can be characterised as facilitating and supporting transition processes as opposed to gap-filling (providing remediation for students with deficits in competencies, skills, and knowledge), is increasingly promoted in other countries as well, such as the UK (David 2010) and Australia (Catterall et al. 2014). To enable this high level of student preparation, cooperation between the particular educational organisations is crucial. In this regard, Jäppinen and Maunonen-Eskelinen (2012) stressed the importance of collaborative practices of the personnel, or, in other words, distributed pedagogical leadership to guarantee transitional fluency (see also Jäppinen 2010). This seems to be a crucial condition for the effectiveness of continuing learning pathways, such as the GL. In the GL, cooperation between the various educational levels was realized as one of the pillars of the educational innovation (which was relatively easy in this case since the original vmbo and mbo programmes were offered by the same VET institutes) and this cooperation concerned both planning and implementation of the learning pathway (see also Biemans et al. 2013).

At this moment, however, it should be noted that there are only first -but promisingindications of the effectiveness of the GL as continuing learning pathways. Moreover, the specific target group of the GL consists of high performing students. In this regard, differences exist between various continuing learning pathways in vocational education: Some pathways are especially designed for high performing students (see also Jäppinen and Maunonen-Eskelinen 2012), while other routes aim at regular student populations. When interpreting the results of the present study, the specific target group should be taken into account and, at this moment, preliminary conclusions on the effectiveness of learning pathways such as the GL cannot be generalised to other target groups. Future research should provide more insights in this respect. 
In the context of this specific project, future research will focus on: 1) analysis of students' transitions in the different learning pathways in the second phase of their trajectories; 2) analysis of students' learning performance in the second phase of these learning pathways; and 3) analysis of students' experiences, learning performance, and transitions in higher vocational education. Especially the answer to this last question (do the GL lead to more student satisfaction, higher learning performance, and lower drop-out rates in higher vocational education than the traditional pathways?) will be the proof of the pudding since this research would shed light on the longer-term effects of these continuing learning pathways.

Open Access This article is distributed under the terms of the Creative Commons Attribution 4.0 International License (http://creativecommons.org/licenses/by/4.0/), which permits unrestricted use, distribution, and reproduction in any medium, provided you give appropriate credit to the original author(s) and the source, provide a link to the Creative Commons license, and indicate if changes were made.

\section{References}

AOC Council (2012). Sector plan AOCs 2012-2015 sempervivum. Ede: AOC Council.

Bathmaker, A. M., \& Thomas, W. (2009). Positioning themselves: An exploration of the nature and meaning of transitions in the context of dual sector FE/HE institutions in England. Journal of Further and Higher Education, 33(2), 119-130.

Biemans, H., Nieuwenhuis, L., Poell, R., Mulder, M., \& Wesselink, R. (2004). Competence-based VET in The Netherlands: Background and pitfalls. Journal of Vocational Education and Training, 56(4), 523-538.

Biemans, H., Wesselink, R., Gulikers, J., Schaafsma, S., Verstegen, J., \& Mulder, M. (2009). Towards competence-based VET: Dealing with the pitfalls. Journal of Vocational Education and Training, 61(3), 267-286.

Biemans, H.J.A., De Bruijn, E., Den Boer, P.R., \& Teurlings, C.C.J. (2013). Differences in design format and powerful learning environment characteristics of continuing pathways in vocational education as related to student performance and satisfaction. Journal of Vocational Education and Training, 65(1), 108-126.

Billett, S. (2001). Knowing in practice: Re-conceptualising vocational expertise. Learning and Instruction, 11, 431-452.

Bloomer, M., \& Hodkinson, P. (2000a). The complexity and unpredictability of young people's learning careers. Education and Training, 2(2), 68-74.

Bloomer, M., \& Hodkinson, P. (2000b). Learning careers: Continuity and change in young people's dispositions to learning. British Educational Research Journal, 26(5), 507-583.

Bradley, D. (2008). Review of Australian higher education: Final report. Canberra: Commonwealth of Australia.

Brockmann, M., Clarke, L., Méhout, P., \& Winch, C. (2008). Competence-based vocational education and training (VET): The cases of England and France in a European perspective. Vocations and Learning: Studies in Vocational and Professional Education, 1, 227-244.

Catterall, J., Davis, J., \& Yang, D. F. (2014). Facilitating the learning journey from vocational education and training to higher education. Higher Education Research \& Development, 33(2), 242-255.

Clarke, L., \& Winch, C. (2007). Vocational Education: International Approaches, Developments and Systems. London/New York: Routledge.

David, M. (Ed.) (2010). Improving learning by widening participation in higher education. London: Routledge.

De Bruijn, E., \& Leeman, Y. (2011). Authentic and self-directed learning in vocational education: Challenges to vocational educators. Teaching And Teacher Education, 27, 694-702.

Dutch Educational Council (2014). Overgangen in het onderwijs [transitions in education]. The Hague: Dutch Educational Council.

Dutch Ministry of Education, Culture, and Science (2005). Vmbo: het betere werk [pre-vocational secondary education (vmbo): work of higher quality]. The Hague: Ministry of Education, Culture, and Science.

European Commission (2008). The European qualification framework for lifelong learning (EQF). Luxembourg: Office for Official Publications of the European Communities. 
Gorard, S., Smith, E., May, H., Thomas, L., Adnett, N., \& Slack, K. (2006). Review of widening participation research: Addressing the barriers to participation in higher education. A report to HEFCE. York: University of York, Higher Education Academy and Institute for Access Studies.

Griffiths, T., \& Guile, D. (2003). A connective model of learning: The implications for work process knowledge. European Educational Research Journal, 2(1), 56-73.

Harris, R., \& Rainey, L. (2012). Learning pathways between and within vocational and higher education: Towards a typology? Australian Educational Researcher, 39, 107-123.

Harris, R., Sumner, R., \& Rainey, L. (2005). Student traffic: Two-way movement between vocational education and training and higher education. Adelaide: National Centre for Vocational Education Research.

Hayward, G. (2006). Participation, progression and success in vocational learning: A quantitative analysis of system performance. London: Learning and Skills Research Centre.

Hoelscher, M., Hayward, G., Ertl, H., \& Dunbar-Goddet, H. (2008). The transition from vocational education and training to higher education: A successful pathway? Research Papers in Education, 23(2), 139-151.

Jäppinen, A.-K. (2010). Preventing early leaving in VET: Distributed pedagogical leadership in characterizing five types of successful organizations. Journal of Vocational Education \& Training, 62(3), 297-312.

Jäppinen, A.-K., \& Maunonen-Eskelinen, I. (2012). Organisational transition challenges in the Finnish vocational education - Perspective of distributed pedagogical leadership. Educational Studies, 38(1), 39-50.

Keeley, B. (2007). Human capital. how what you know shapes your life. Paris: OECD.

Koopman, M., Teune, P., \& Beijaard, D. (2011). Development of student knowledge in competence-based prevocational secondary education. Learning Environments Research, 14(3), 205-227.

Krause, K., \& Coates, H. (2008). Students' engagement in first-year university. Assessment and Evaluation in Higher Education, 33(5), 493-505.

Kuijpers M.A.C.T. (2014). Doorstroom vmbo-mbo [Transition pre-vocational secondary education (vmbo) secondary vocational education (mbo)]. NRO Call for proposals Green Education Research. Den Haag: Dutch Organisation for Scientific Research (NWO).

Leese, M. (2010). Bridging the gap. Journal of Further and Higher Education, 34(2), 239-251.

OECD (2010). Education of a glance. Paris: OECD.

Putnam, R. T., \& Borko, H. (2000). What do new views of knowledge and thinking have to say about research on teacher learning? Educational Researcher, 29, 4-15.

Sturing, L., Biemans, H.J.A., Mulder, M., \& De Bruijn, E. (2011). The nature of study programmes in Vocational Education: evaluation of the model for Comprehensive Competence-Based Vocational Education in The Netherlands. Vocations and Learning: Studies in Vocational and Professional Education, 4(3), 191-210.

Van den Berg N. (2013). Doorstroom tussen groen vmbo en mbo. Onderzoek naar relevante factoren en sturingsmogelijkheden [transition between agricultural pre-vocational secondary education and secondary vocational education. research on relevant factors and possibilities for governance]. Ede/Rotterdam: AOC Council/Strix Aluco-Research \& Innovation.

Watson, D. (2006). New labour and higher education. Perspectives: Policy and Practice in Higher Education, 10, 92-96.

Wesselink, R., De Jong, C., \& Biemans, H.J.A. (2010a). Aspects of competence-based education as footholds to improve the connectivity between learning in school and in the workplace. Vocations and Learning: Studies in Vocational and Professional Education, 3(1), 19-38.

Wesselink, R., Dekker-Groen, A.M., Biemans, H.J.A., \& Mulder, M. (2010b). Using an instrument to analyse competence-based study programmes; Experiences of teachers in Dutch vocational education and training. Journal of Curriculum Studies, 42(6), 813-829.

Wheelahan, L. (2008). Neither fish nor fowl: The contradiction at the heart of Australian tertiary education. Journal of Access Policy and Practice, 5(2), 133-152.

Winters, A. (2012). Career learning in vocational education: Guiding conversations for career development. Leuven: KU Leuven.

Dr. Harm Biemans is associate professor at the Education and Competence Studies group of Wageningen University. His research concentrates on competence development of (future) professionals and design and effects of corresponding learning environments.

Ing. Hans Mariën is researcher at IVA Education, a research institute at Tilburg University with a focus in the field of educational research. His research interests focus on school-to-work transition (mainly in higher education) and learning performance in different levels of education. 
Erik Fleur M.A. is researcher for the Dutch Department of Education, Culture \& Science. His research interests focus on transitions within the educational system and the relations between vocational and higher education.

Dr. Hilde Tobi is associate professor research methodology at Wageningen University. Her research interests focus on methodology and measurement for interdisciplinary research.

Prof. Dr. Loek Nieuwenhuis is professor of education at the HAN University of Applied Sciences and the Open University of The Netherlands. His research topics concern pedagogy of the professions and vocational education.

Dr. Piety Runhaar is assistant professor at the Education and Competence Studies group of Wageningen University. Her research concentrates on professional development of VET teachers and related human resource management. 